\title{
CANCER PHARMACOGENETICS: PERSPECTIVE ON NEWLY DISCOVERED AND IMPLEMENTED PREDICTIVE BIOMARKERS
}

\author{
R. Roncato ${ }^{1}$, E. Cecchin ${ }^{1}$, C. Dalle Fratte ${ }^{1}$, G. Decorti ${ }^{2,3}$, M. Del Re ${ }^{4}$, R. Franca ${ }^{3}$, \\ S. Nobili ${ }^{5}$, G. Ravegnini ${ }^{6}$, G. Stocco ${ }^{7}$, E. Mini ${ }^{8}$, G. Toffoli ${ }^{1}$, Cancer Pharmacology \\ Working Group of the Italian Society of Pharmacology (CPWG-SIF)
}

${ }^{1}$ Experimental \& Clinical Pharmacology Unit, Centro di Riferimento Oncologico di Aviano (CRO), IRCCS, Aviano, Italy ${ }^{2}$ Institute for Maternal and Child Health-IRCCS Burlo Garofolo, Trieste, Italy

${ }^{3}$ Department of Medical, Surgical and Health Sciences, University of Trieste, Trieste, Italy

${ }^{4}$ Department of Clinical and Experimental Medicine, Unit of Clinical Pharmacology and Pharmacogenetics, University of Pisa, Pisa, Italy

${ }^{5}$ Department of Neurosciences, Imaging and Clinical Sciences, G. d'Annunzio University, Chieti-Pescara, Italy ${ }^{6}$ Department of Pharmacy and Biotechnology, Alma Mater Studiorum-University of Bologna, Bologna, Italy ${ }^{7}$ Department of Life Sciences, University of Trieste, Trieste, Italy

${ }^{8}$ Department of Health Science, Section of Clinical Pharmacology and Oncology, University of Florence, Florence, Italy

E-mail: gtoffoli@cro.it

Doi: 10.36118/pharmadvances.2021.03

\section{SUMMARY}

Implementation of pharmacogenetics (PGX) in the clinical practice of cancer therapy is one of the goals for a precision medicine. For some anticancer drugs (fluoropyrimidines and irinotecan) precise recommendations by regulatory agencies are available. However, in the future, implementation of PGx in clinical practice should also consider the anticancer drugs without available pharmacogenetic guidelines and the increasingly important role of rare PGx variants. To overcome barriers to PGx implementation we need to focus on pharmacogenetic test clinical utility by adopting a panel-based approach and sensitize both physician and patients to the therapeutic value of pharmacogenetic tests.

\section{Key words}

Implementation; pharmacogenetics; rare variants; guidelines; biomarker.

\begin{abstract}
Impact statement
Implementation of Pharmacogenetics in Oncology is mandatory for precision medicine. Validated pharmacogenetic biomarkers require a pre-therapeutic screening, while the contribute of less validated and rare variants, of polytherapeutic and polygenic effect on patient's phenotype are becoming increasingly important.
\end{abstract}

istics of patients. PGx has grown rapidly in the last two decades and PGx biomarkers are now available for several drugs to the point that regulatory agencies (US Food and Drug 
Administration (FDA) and European Medical Agency (EMA)) incorporated PGx information into drug labels (1).

In cancer therapy, PGx has a peculiar role since many anticancer drugs are characterized by a narrow therapeutic index and the genetic background of patients can affect the absorption, distribution, metabolism, and elimination (ADME) of drugs. Regardless of the recommendations made by regulatory agencies on anticancer drug use, only for a few of them a pretherapeutic pharmacogenetic screening is considered mandatory in the clinical care (as for fluoropyrimidines, irinotecan) (see table I). Advances in genetics are changing and next-generation sequencing (NGS) of DNA offers novel ways to identify PGx factors. Rare genetic variants represent new PGx biomarker frontier to be investigated and elucidated in their contribution to interindividual variability (2). In addition, PGx is acquiring a pivotal role to better define not only the simple interaction between a candidate gene and a specific drug, but also polytherapy that is the basis of all the anticancer regimens. In this contest, the gene activity related to one genetic variant can affect the metabolism of more than one drug concomitantly administered with multiple genes and drug interactions involvement (3). Analogously, the activity of a sin- gle drug can be affected by genetic variants in more than one gene acting at a different point of the metabolic pathway of the drug. In this review we focus on PGx implementation in cancer therapy.

\section{NATIONAL AND INTERNATIONAL GUIDELINES}

Among the most relevant enabling tools in the germline PGx implementation process, clinical guidelines for the application of validated PGx markers have acquired a recognized role in the everyday management of patients' treatment (4). Current PGx guidelines propose dosing/ treatment personalization for a set of gene-drug pairs in oncology that shows substantial evidence linking genotype and clinical phenotype.

Most of the known PGx information is shared through PharmGKB (www.pharmgkb.org) (1, 5) an open-source literature database, curated and continuously updated by an international panel of experts committed to implement the translation of $\mathrm{PG} x$ recommendations into the clinical practice. PharmGKB collects the PGx guidelines promoted for the first time by the Clinical Pharmacogenetics Implementation Consortium (CPIC) and the Dutch Pharmacogenetics Working Group (DPWG), two

Table I. Recommendations for main pharmacogenetic biomarkers available in anticancer treatments.

\begin{tabular}{|c|c|c|c|c|c|c|}
\hline \multirow[b]{2}{*}{ Gene-drug couple } & \multicolumn{2}{|c|}{ Regulatory agencies } & \multicolumn{3}{|c|}{ Scientific Societies } & \multirow[t]{2}{*}{ PharmGKB } \\
\hline & EMA & FDA & DPWG & SIF-AIOM & CPIC & \\
\hline $\begin{array}{l}\text { DPYD- } \\
\text { Fluoropyrimidines }\end{array}$ & $\begin{array}{l}\text { Testing } \\
\text { required }\end{array}$ & $\begin{array}{l}\text { Actionable } \\
\text { PGx }\end{array}$ & $\begin{array}{l}\text { Testing } \\
\text { required }\end{array}$ & $\begin{array}{l}\text { Testing } \\
\text { required }\end{array}$ & $\begin{array}{l}\text { Testing } \\
\text { required }\end{array}$ & $1 \mathrm{~A}$ \\
\hline UGT1A1-Irinotecan & $\begin{array}{l}\text { Actionable } \\
\text { PGx }\end{array}$ & $\begin{array}{l}\text { Actionable } \\
\text { PGx }\end{array}$ & $\begin{array}{l}\text { Testing } \\
\text { required }\end{array}$ & $\begin{array}{l}\text { Testing } \\
\text { required }\end{array}$ & N.A. & $1 \mathrm{~A}$ \\
\hline CYP2D6-Tamoxifen & N.A. & $\begin{array}{l}\text { Actionable } \\
\text { PGx }\end{array}$ & $\begin{array}{l}\text { Testing } \\
\text { required }\end{array}$ & N.A. & $\begin{array}{l}\text { Testing } \\
\text { required }\end{array}$ & $1 \mathrm{~A}$ \\
\hline $\begin{array}{l}\text { TPMT/NUDT15- } \\
\text { Thiopurines }\end{array}$ & N.A. & $\begin{array}{l}\text { Testing } \\
\text { required }\end{array}$ & $\begin{array}{l}\text { Testing } \\
\text { required }\end{array}$ & N.A. & $\begin{array}{l}\text { Testing } \\
\text { required }\end{array}$ & $1 \mathrm{~A}$ \\
\hline
\end{tabular}

Testing required has been reported when a pharmacogenetic test is recommended; actionable PGx when only information regarding treatment outcome (toxicity and efficacy) in relation to the PGx biomarker have been reported. PGx biomarker considered only informative is not listed for the presented pharmacogenes. N.A. was reported when no PGx guidelines were available. PharmGKB level of evidence 1 A refers to "variant-drug combinations that have variant-specific prescribing guidance available in a current clinical guideline annotation or an FDA-approved drug label annotation" (1). 
international working groups that pioneered implementation of PGx guidelines based on clinical evidence. More recently PGx guidelines from other professional societies such as the Canadian Pharmacogenomics Network for Drug Safety (CPNDS) and the French National Network of Pharmacogenetics (RNPGx) have been included.

Just a few gene-drug couples present validated germline PGx guidelines in oncology, i.e. DPYD-fluoropyrimidines, CYP2D6-tamoxifen, TPMT/NUDT15-thiopurines, and UGT1A1-irinotecan (the latter just for the DPWG). In Italy, a collaboration between the Italian Society of Pharmacology (SIF) and the Italian Society of Medical Oncology (AIOM) was set up to develop and curate $\mathrm{PGx}$ recommendations for fluoropyrimidines and irinotecan (table I).

DPD represents the rate limiting enzyme involved in the catabolism of the anticancer drugs 5-fluorouracil, capecitabine and tegafur. Four variants in DPYD (DPYD*2A, DPYD*13, $D P Y D \_c .2846 C>T$, and DPYD-HapB3) have a validated functional effect in reducing DPD functionality and increasing the risk of severe toxicity related to fluoropyrimidines administration. All the international guidelines now agree that a pre-treatment DPYD genotyping is recommended $(6,7)$, and recently also EMA published a document recommending the DPD screening in order to increase treatment safety that was embraced also by the Italian drug agency (AIFA) (8). Based on the current guidelines, patients that are carriers of one variant allele within the panel should receive a halved starting dose of fluoropyrimidines. In the remote possibility that a patient should carry more than one variant allele, patients should be phenotyped (i.e. DPD activity should be determined) or otherwise fluoropyrimidines treatment should be avoided. Italian SIF-AIOM recommendation also include a warning on the missense DPYD c. $2194 G>A\left(D P Y D^{*} 6\right)$ that could be considered as a post-toxicity analysis in absence of the four validated variants. However, the variant has not been judged sufficiently powered to be included in other PGx guidelines or to be recommended by regulatory agencies as a pre-treatment test.

Irinotecan is converted into its active metabolite SN-38, which in turn is detoxified by the UDP-glucuronosyltransferase (UGT) enzyme encoded by the UGT1A1 gene. The risk of irinotecan toxicity increases in presence of genetic variants associated with a reduced UGT1A1 enzyme activity, such as UGT1A1*28. The DPWG as well the SIF-AIOM recommendations suggest a starting dose of $70 \%$ of the standard dose for homozygous carriers of the UGT1A1*28 allele, while no precautionary starting dose reduction is needed for heterozygous carriers of the UGT1A $1 * 28$ allele (9). These guidelines are currently not endorsed by national or international regulatory agencies.

Thiopurines (azathioprine, mercaptopurine, thioguanine) are inactivated and detoxified by the enzymes TPMT and NUDT15, whose activity is affected by the presence of single nucleotide polymorphisms in the corresponding genes, which might lead to drug accumulation and toxicity. Dosing recommendations for thiopurines based on TPMT and NUDT15 genotype have been published by the CPIC and the DPWG $(10,11)$. Both the guidelines recommend substantial dose reductions for individuals who have low or deficient enzyme activity, including considering an alternative drug to thiopurines.

Tamoxifen is activated by CYP2D6 into hydroxylated metabolites having greater antiestrogenic potency than the parent drug. Patients bearing loss-of-function genetic polymorphisms on CYP2D6 have lower exposure to active tamoxifen metabolites concentrations and could therefore be exposed to the risk of under-treatment and disease recurrence. Dosing recommendations for tamoxifen based on $\mathrm{CY}$ P2D6 genotype have been published by the CPIC and the DPWG $(12,13)$. Both the guidelines recommend that an alternative medication should be considered for patients who are CYP2D6 intermediate metabolizers or poor 
metabolizers. Alternatively, a dose increase can be considered for these patients coupled with monitoring of endoxifen plasma level.

\section{STATE OF ART ON ANTI-TUMOUR DRUG PHARMACOGENETICS WITHOUT AVAILABLE PHARMACOGENETIC GUIDELINES}

In addition to the above reported drugs/pharmacogenes associations, other less validated genetic polymorphisms have been associated with response to anticancer agents, either cytotoxic drugs or targeted agents. However, for most of these associations the current level of evidence is low and thus a pre-therapeutic genetic test is considered only informative by FDA and/or EMA. For some of these drugs, recommendation guidelines by CPIC, DPWG or other international working groups (i.e. The CPNDS) integrate information present in drug labels by extending data also to other genes potentially involved in treatment response variations. An example is represented by cisplatin and TPMT variants. FDA considers a TPMT genetic test only informative in relation to the prediction of cisplatin-induced ototoxicity in children. In agreement with FDA, CPNDS indicates any non-functional TPMT variant $\left(T P M T^{\star} 2, * 3 \mathrm{~A}, * 3 \mathrm{~B}\right.$ and $\left.{ }^{*} 3 \mathrm{C}\right)$ at high risk for ototoxicity providing a level C - optional recommendation. In addition, CPNDS recommends a pharmacogenetic test of the same TPMT variants for the prevention of cisplatin-induced hearing loss in children with a level A - strong recommendation (14).

CPNDS also published guidelines for polymorphisms in three genes, RARG, SLC8A3 and UG$T 1 A 6$, in case of pediatric use of doxorubicin and daunorubicin to reduce the incidence of cardiac toxicity. In particular, a level B - moderate recommendation has been provided for the study of RARG rs2229774, SLC28A3 rs7853758 and UGT1A6 rs17863783 variants (15).

For other cytotoxic agents (i.e. paclitaxel, docetaxel, vincristine, oxaliplatin), associations with polymorphisms in genes relevant for their mechanisms of action or resistance (i.e. ABCB1 variants), have been reported (1618). Polymorphisms in genes encoding detoxifying or metabolic enzymes have also been associated with a lower response to oxaliplatin (i.e. GSTP1 variants) $(18,19)$ and cyclophosphamide (i.e. CYP2B6 variants) (17) as well as with a potential high risk of paclitaxel induced peripheral neuropathy (i.e. CYP3A4 variants) (20). However, current available data do not support evidence for recommending genetic test before the administration of these drugs and the international pharmacogenetic working groups did not propose specific guidelines. An exception is represented by the RNPGx that suggests MTHFR and SLCO1B1 variants as potential biomarkers of methotrexate toxicity, although providing no recommendation essentially based on controversial results (mainly for MTHFR) (21).

Regarding targeted therapy, evidence related to genetic variants associated with toxicity or clinical outcome are reported as actionable or informative PGx, which represent the lower level of evidence.

Most examples are associated with metabolism enzymes. Considering the CYP family, CYP2C9 and CYP2D6 have been described as involved in toxicity related events for the tyrosine kinase inhibitors (TKI) of FGFR1-4, erdafitinib, and the EGFR TKI, gefitinib, respectively. CYP2C9 poor metabolizers $(* 3 / * 3)$ may result in higher systemic concentrations and higher adverse reaction risk; for this reason, these patients should be monitored for adverse reactions (22). Similarly, CYP2D6 poor metabolizers in treatment with gefitinib should be carefully monitored even if dose adjustment is not required (23).

Another gene involved in metabolism and often associated with PGx toxicity is UGT1A1. Many drugs are metabolized by the UGT1 family enzymes, however, the isoform $1 \mathrm{~A} 1$ is the most frequently related to adverse events. On PharmGKB platform, many examples are reported. Among these, UGT1A1 genotype is important in patients under sacituzumab 
govitecan-hziy treatment. This drug is an antibody-drug conjugate that integrates a humanized monoclonal antibody, which targets the human trophoblast cell-surface antigen 2 (Trop-2), with SN-38 (the active metabolite of irinotecan) to promote the delivery of high concentrations of $\mathrm{SN}-38$ to tumors. As for irinotecan, the risk of toxicity increases in presence of genetic variants associated with a reduced UGT1A1 enzyme activity, such as UGT1A1*28 (24).

Similarly, nilotinib, a TKI used for chronic myeloid leukemia, can increase bilirubin levels since UGT1A1 glucuronidates bilirubin for elimination. Even in this case, poor metabolizers UGT1A $1 * 28$ have a higher risk of hyperbilirubinemia (25). A similar mechanism has been also observed for pazopanib, a TKI for patients with advanced renal cell carcinoma. UGT1A $1 * 28$ patients should be monitored for higher adverse reaction risk (26). The same drug treatment can promote deregulation of liver enzymes levels in patients carrying the HLB*5701 variant and should carefully followed-up (27). This specific variant is well characterized for its relationship with the abacavir hypersensitivity adverse event, but the mechanism is not completely understood yet.

An additional example of PGx that deserves to be mentioned is dabrafenib and glucose-6-phosphate dehydrogenase (G6PD), a cytoplasmic enzyme that catalyzes the first and rate-limiting step of the pentose phosphate pathway (28). Dabrafenib, which contains a sulfonamide moiety, confers a potential risk of hemolytic anemia in patients with G6PD deficiency. Even in this case, it is suggested to monitor patients with G6PD deficiency for signs of hemolytic anemia; FDA has included this indication within the dabrafenib drug label.

\section{ROLE OF RARE VARIANTS IN IMPROVING THE SENSITIVITY OF PHARMACOGENETIC TESTS}

In recent years, NGS high-throughput technologies have allowed better insights of genomic
DNA, deepening our knowledge of inter-individual genetic variation with large-scale studies and shedding light on the contribution of rare variants (MAF $<1 \%$ ) on pharmacological phenotypes (29). Few studies have considered the impact of application of NGS approaches on pharmacogenes.

One of the first studies evaluated SLCO1B1 transporter gene rare damaging non-synonymous variants and their effect on methotrexate exposure after high-doses infusions in 699 children affected by acute lymphoblastic leukemia. Inter-individual variation in methotrexate clearance resulted in important changes in the anti-leukemic effectiveness and in the occurrence of severe drug-related gastrointestinal and hematological toxicities. SLCO1B1 rare variants were studied as follow-up of a pharmacogenetic GWAS anaysis (30): they contributed for $17.8 \%$ of SLCO1B1's effects on drug clearance $1.9 \%$ of total variation), delaying drug elimination with larger effect sizes than common SNPs (31).

Recently, in a more comprehensive study, using exome sequencing data from 60,706 unrelated individuals, Ingelman-Sundberg and coworkers investigated the variability in 208 pharmacogenes, clinically relevant for their role in drug ADME as well as in drug pharmacodynamics. They found that each individual harbored 40.6 putatively functional variants on average, $10.8 \%$ being rare, and that the pharmacological relevance of these rare mutations was highly drug-specific, depending on the gene products involved. Authors analyzed five candidate drugs more in detail to assess whether rare genetic variants account for a substantial part of the unexplained inter-individual differences in drug metabolism phenotype. In this analysis it emerged that sequencing of candidate transporter genes (SLCO1B1, ABCC1, ABCB1, ABCC2, ABCG2) would likely improve management of chemotherapy with irinotecan to prevent toxicity, since $8.7 \%$ of all deleterious variants found in SLCO1B1, 40.5\% in $A B C C 1$, and $100 \%$ in $A B C B 1, A B C C 2, A B C G 2$ genes were rare, accounting for $>40 \%$ of the still unexplained in- 
ter-individual variability in irinotecan transport. For other drugs such as voriconazole, rare variants only contributed less than $10 \%$ of the key metabolic and/or transport processes (32). Integrating rare genetic variants into pre-emptive pharmacogenetic tests will allow genotyping to be highly effective for a true individualization of patients' drug treatment regimens (33). Besides understanding which drug would fully benefit from a pharmacogene characterization at the sequencing level, population-specific patterns of rare variants should be known $(34,35)$ and taken into consideration for developing population-adjusted genetic profiling strategies $(2,36,37)$. For instance, CPIC guidelines recommended TPMT and NUDT15 genotyping of common variants for thiopurine dosing. NUDT15 risk alleles explained the majority of thiopurine-induced hematological toxicity in Asians; targeted sequencing of the full-length NUDT15 gene could thus be a reasonable integrative approach to carefully predict the initial dosage in Asiatic patients (38). In other populations, such as in the Europeans, the characterization of variants in TPMT should be a priority (39).

Cost-effectiveness of the analysis of rare variants in pharmacogenes has still to be clearly estimated, however NGS costs have been consistently reducing in recent years and this approach is promising for the future pharmacogenomic analysis (36).

\section{BARRIERS TO PGX IMPLEMENTATION AND RELATED PROJECTS}

Despite the clinical evidence for PGx testing, its translation into clinical practice is slow and

\section{Issues and challenges}

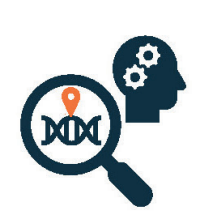

PGx biomarker discovery

- Specific clinical context of application;

- low prevalence polymorphism and rare variants;

- high-throughput technologies;

- analytical validation (assay development and standardization).

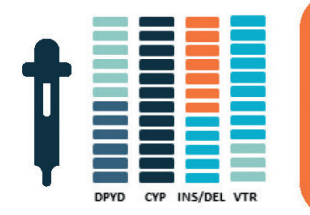

\section{Clinical research}

- Clinical validity assessment (sample size, clinical follow-up, evidence for

clinical relevance);

- clincal utility assessment (test-cost-effectiveness and cost-consequence).

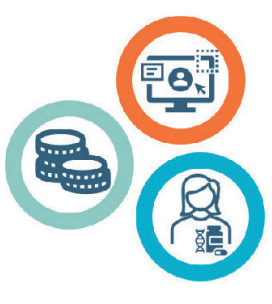

\section{Clinical practice}

- Integration in clinical workflow (EHR and CDSS);

- improvement of PGx knowledge and education

- improvement of clinical utility (single gene vs panel-based approach);

- PGx results interpretation and treatment personalization;

- PGx test reimbursement;

- PGx information in drug leaflet by regulatory agencies;

- availability of PGx guidelines from specific scientific societies;

- integration with polytherapeutic and polygenic effects.

Figure 1. Issue and challenges from the discovery to clinical practice of pharmacogenetic biomarker. The figure represents all the existed barriers and challenges to each step of PGx biomarker development from research to clinical practice. 
hindered by many barriers that prevent its implementation on a larger scale (figure 1). Such translation requires, beyond an established statistical association with an outcome of drug therapy, evidence of clinical-utility and cost-effectiveness that will likely result in favorable coverage decisions from payers (40). Lack of a unique clinical utility definition results in uncertainty regarding the level of evidence required to support implementation of a PGx test into the clinic. According to some authors, the recommendation for testing can be "potentially beneficial", "beneficial" and "essential" depending upon both the clinical effects associated with the gene-drug interaction and the level of evidence, as assessment of test sensitivity and specificity, supporting the associated clinical effect (41). Another barrier for PGx test implementation includes reimbursement issues, especially in those cases in which there is a relatively low allele frequency of deficient variants, as in the DPYD case. Low allele frequencies are inversely related to the incremental benefit of screening, with consequences on the cost-effectiveness assessment of the PGx test. This results in uncertainty about level of coverage by insurance and reimbursement. Regardless, reluctance to embrace PGx testing is often the result both of unfamiliarity with PGx knowledge and of the physician's crucial position in PGx-based prescription delivery. Indeed, although physician may perceive the benefit of using PGx, they are in a front-line position to handle the potential volume of such information by reviewing, interpreting, delivering PGx test results and providing follow-up to the patient. Moreover, pharmacogenomic knowledge will further increase in the future considering the continuing advances in NGS which will result in new drug-gene interactions discovered. Eventually, to make economically feasible in community-based practice settings to utilize genetics to guide drug prescribing, structured approaches (i.e. algorithms) and implementation of point-of-care electronic clinical decision support (CDS) are most needed (42).
Clinical PGx programs utilizing implementation models have been reported since the early 2000s and continue to be published in a variety of healthcare settings including academic health centers, primary care and community practice adopting both preemptive and point-of-care testing (43). The eMERGEPgx is a partnership of Electronic Medical Records and Genomics Network (eMERGE) and the Pharmacogenomics Research Network (PGRN) aimed at integrating clinically validated pharmacogenetic genotypes into the electronic health record and associated CDS (44). A few medical institutions also started implementation projects to overcome reactive genotyping in favour of preemptive genotyping. This is the case of the Icahn School of Medicine at Moun Sinai with CLIPMERGE, the Mayo Clinic with RIGHT project and the Vanderbilt University Medical Center with PREDICT. Mount Sinai Medical Center's, member of eMERGE network, initiated the CLIPMERGE PGx program directed at optimizing the use of $P G x$ in clinical care by using a DNA biobank derived cohort (BioMe). Another main network involved in the integration of patient's genetic data into their clinical care is IGNITE, involving the University of Florida's with the PGx implementation program Personalized Medicine Program, the Indiana University's with INGENIOUS program and the Vanderbilt University's with I3P program. Cleveland Clinic's Personalized Medication Program, the St. Jude Children's Research Hospital's PG4KDS program, the University of Chicago's 1200 Patient Project represented other initial efforts to translate $P G x$ in clinical care (45). A multicentric prospective, controlled, block-randomized clinical trial (PREemptive Pharmacogenomic testing for prevention of Adverse drug Reactions, PREPARE) initiated in 7 European countries including Italy with the financial support of the European Community Horizon 2020 recently concluded. The aim of the PREPARE study was to assess the clinical utility of implementing a panel of PGx markers into routine care on the prevention of toxic events, on the improve- 
ment of patients' quality of life and on the reduction of toxicity-related costs (46). This huge international effort will hopefully demonstrate that PGx implementation in clinical practice is feasible and whether a pre-treatment PGx approach will positively impact health outcomes, decision making and costs in several field of Pharmacology, including cancer.

\section{CONCLUSIONS}

Implementation of PGx in oncology remains an important goal for the personalization of therapy. The recommendations of the regulatory agencies, although limited to only a few antineoplastic drugs, generate important evidence for its clinical utility in support of implementation in the clinical care. However, today we are probably facing a decisive point in PGx research, where we acknowledge that the initial historical search for variants in candidate genes did not produce the expected breakthrough change in the prescription of oncologic drugs. Indeed, the effect of a drug follows a multimodal network at the interface between many interactions between drugs and genes. The activity of a single variant in a single gene has only in extreme cases (i.e. in the case of ${ }^{*} 2 A$ or ${ }^{*} 13$ variants of DYPD) a dramatic effect. The activity of a gene can depend on several variants located on the same actionable gene which can be present in different combinations predicting different phenotypes. The implementation of a panel-based pharmacogenetic test covering all the actionable genes available in a pre-therapeutic setting has been referred as the PGx intervention with the most clinical impact (47). Information on other pharmacogenes can be widely exploited in the oncologic context considering the polytherapeutic nature of most regimens. Drugs can interact each other and their action is the result of a polygenic effect depending on the functional activity of several genes. On this ground, focusing on gene-gene interactions and between multiple genes and multi- ple drugs could represent a PGx strategy with a real clinical relevance. Finally, emerging observation seem to indicate that rare variants in actionable genes could have a significant pharmacological effect which has required, in some instance, an NGS diagnostic approach to unravel the complexity of the patient's developed phenotype. Even the variant germline burden has been considered to be of potential impact on drug effect as suggested by Lauschke et al. (48) where the adoption of artificial intelligence strategy will play an increasingly important role. This raises the problem of introducing expensive and more time-consuming technologies into clinical practice also considering the high level of personnel specialization required to conduct and interpret these data. It is mandatory to carefully evaluate the feasibility of such approaches, probably not scalable in the short-term, in terms not only of costs but also of clinical utility.

Ultimately, the implementation of PGx in clinical practice cannot overlook the aspects of education and sensitization that are still lacking among both healthcare professionals and patients (49). If these aspects are not given the attention they deserve, the lifetime benefit of pharmacogenetic panel test on patient clinical outcome is reduced to a pre-therapeutic diagnostic exam, losing the therapeutic opportunity to be used for future prescriptions. PGx might take many years to be implemented considering that, although sequencing techniques are increasingly accessible, they require specialized personnel and prolonged turnaround times, resulting in a high implementation cost in the realities of clinical oncological practice. In this scenario, the implementation projects carried out at national and international level acquire a crucial importance to tackle such challenges.

\section{CONFLICT OF INTERESTS}

The authors declare that they have no conflict of interests. 


\section{REFERENCES}

1. The Pharmacogenomics Knowledge Base (Pharmgkb). Available at: https://www. pharmgkb.org/. Accessed July 27, 2016.

2. Kozyra M, Ingelman-Sundberg M, Lauschke VM. Rare genetic variants in cellular transporters, metabolic enzymes, and nuclear receptors can be important determinants of interindividual differences in drug response. Genet Med 2016. Doi:10.1038/ gim.2016.33.

3. Malki MA, Pearson ER. Drug-drug-gene interactions and adverse drug reactions. Pharmacogenomics J 2020;20(3):355-66. Doi:10.1038/s41397-019-0122-0

4. Bank PCD, Swen JJ, Guchelaar H-J. Implementation of Pharmacogenomics in Everyday Clinical Settings. In: Advances in Pharmacol, Elsevier 2018;83:219-46. Doi:10.1016/bs.apha.2018.04.003.

5. Pharmacogenomics Knowledge for Personalized Medicine. Available at: https://ascpt.onlinelibrary.wiley.com/doi/ epdf/10.1038/clpt.2012.96. Accessed March 30, 2021.

6. Amstutz $U$, Henricks LM, Offer SM, et al. Clinical Pharmacogenetics Implementation Consortium (CPIC) Guideline for Dihydropyrimidine Dehydrogenase Genotype and Fluoropyrimidine Dosing: 2017 Update. Clin Pharmacol Ther 2018;103(2):210-6. Doi:10.1002/cpt.911.

7. Lunenburg CATC, van der Wouden $\mathrm{CH}_{\text {, }}$ Nijenhuis $M$, et al. Dutch Pharmacogenetics Working Group (DPWG) guideline for the gene-drug interaction of DPYD and fluoropyrimidines. Eur J Human Gen 2020;28(4):508-17. Doi:10.1038/s41431019-0540-0.

8. EMA recommendations on DPD testing prior to treatment with fluorouracil, capecitabine, tegafur and flucytosine. Available at: https://www.ema.europa.eu/ en/documents/referral/fluorouracil-fluorouracil-related-substances-article-31-referral-ema-recommendations-dpd-testing en. Accessed 30 April 2020.
9. Dean L. Irinotecan Therapy and UGT1A1 Genotype. In: Pratt VM, Scott SA, Pirmohamed M, et al. Med Gen Summaries, National Center for Biotechnology Information (US) 2012. Available at: http://www. ncbi.nlm.nih.gov/books/NBK294473/. Accessed March 30, 2021.

10. Relling MV, Schwab M, Whirl-Carrillo $M$, et al. Clinical Pharmacogenetics Implementation Consortium Guideline for Thiopurine Dosing Based on TPMT and NUDT 15 Genotypes: 2018 Update. Clin Pharmacol \& Ther 2019;105(5):1095-105. Doi:10.1002/cpt.1304.

11. Royal Dutch Pharmacists Association (KNMP). Dutch Pharmacogenetics Working Group (DPWG). Pharmacogenetic Guidelines, Netherlands. TPMT: thioguanine March 2021. Available at: https:// www.knmp.nl/patientenzorg/medicatiebewaking/farmacogenetica/ pharmacogenetics-1.

12. Royal Dutch Pharmacists Association (KNMP). Dutch Pharmacogenetics Working Group (DPWG). Pharmacogenetic Guidelines, Netherlands. Tamoxifen - CYP2D6 2017. Available at: http://kennisbank.knmp.nl.

13. Goetz MP, Sangkuhl K, Guchelaar H-J, et al. Clinical Pharmacogenetics Implementation Consortium (CPIC) Guideline for CYP2D6 and Tamoxifen Therapy. Clin Pharmacol \& Ther 2018;103(5):770-7. Doi:10.1002/cpt.1007.

14. Lee JW, Pussegoda K, Rassekh SR, et al. Clinical Practice Recommendations for the Management and Prevention of Cisplatin-Induced Hearing Loss Using Pharmacogenetic Markers. Ther Drug Monit 2016;38(4):423-31. Doi:10.1097/ FTD.0000000000000298.

15. Aminkeng F, Ross CJD, Rassekh SR, et al. Recommendations for genetic testing to reduce the incidence of anthracycline-induced cardiotoxicity. Br J Clin Pharmacol 2016;82(3):683-95. Doi:10.1111/ bcp.13008. 
16. Hlaváč V, Holý P, Souček P. Pharmacogenomics to Predict Tumor Therapy Response: A Focus on ATP-Binding Cassette Transporters and Cytochromes P450. J Pers Med 2020;10(3). Doi:10.3390/ jpm10030108.

17. Carr DF, Turner RM, Pirmohamed M. Pharmacogenomics of anticancer drugs: Personalising the choice and dose to manage drug response. $\mathrm{Br} \mathrm{J}$ Clin Pharmacol 2021;87(2):237-55. Doi:10.1111/bcp.14407.

18. Bahrami A, Khazaei M, Hassanian SM et al. Targeting the tumor microenvironment as a potential therapeutic approach in colorectal cancer: Rational and progress. J Cell Physiol 2018;233(4):2928-36. Doi:10.1002/jcp.26041.

19. Fariña Sarasqueta $A$, van Lijnschoten $G$, Lemmens VEPP, Rutten HJT, van den Brule AJC. Pharmacogenetics of oxaliplatin as adjuvant treatment in colon carcinoma: are single nucleotide polymorphisms in GSTP1, ERCC1, and ERCC2 good predictive markers? Mol Diagn Ther 2011;15(5):277-283. Doi:10.1007/BF03256419.

20. Cliff J, Jorgensen $A L$, Lord $R$, et al. The molecular genetics of chemotherapy-induced peripheral neuropathy: A systematic review and meta-analysis. Crit Rev Oncol Hematol 2017;120:127-140. Doi:10.1016/j. critrevonc.2017.09.009.

21. Quaranta S, Thomas F. Pharmacogenetics of anti-cancer drugs: State of the art and implementation - recommendations of the French National Network of Pharmacogenetics. Therapie 2017;72(2):205-215. Doi:10.1016/j.therap.2017.01.005.

22. Markham A. Erdafitinib: First Global Approval. Drugs 2019;79(9):1017-21. Doi:10.1007/s40265-019-01142-9.

23. Kobayashi H, Sato K, Niioka T, et al. Effects of polymorphisms in CYP2D6 and $A B C$ transporters and side effects induced by gefitinib on the pharmacokinetics of the gefitinib metabolite, O-desmethyl gefitinib. Med Oncol 2016;33(6):57. Doi:10.1007/s12032-016-0773-5.
24. Bardia A, Messersmith WA, Kio EA, et al. Sacituzumab Govitecan, a Trop-2-Directed Antibody-Drug Conjugate, for Patients with Epithelial Cancer: Final Safety and Efficacy Results from the Phase 1/2 IMMU132-01 Basket Trial. Ann Oncol 16, 2021. Doi:10.1016/j.annonc.2021.03.005.

25. Shibata T, Minami Y, Mitsuma A, et al. Association between severe toxicity of nilotinib and UGT1A1 polymorphisms in Japanese patients with chronic myelogenous leukemia. Int J Clin Oncol 2014;19(2):391396. Doi:10.1007/s10147-013-0562-5.

26. Nelson RS, Seligson ND, Bottiglieri S, et al. UGT1A1 Guided Cancer Therapy: Review of the Evidence and Considerations for Clinical Implementation. Cancers (Basel) 2021;13(7). Doi:10.3390/cancers13071566.

27. $\mathrm{Xu} C-F$, Johnson $T$, Wang $X$, et al. HLA-B*57:01 Confers Susceptibility to Pazopanib-Associated Liver Injury in $\mathrm{Pa}$ tients with Cancer. Clin Cancer Res 2016;22(6):1371-77. Doi:10.1158/10780432.CCR-15-2044.

28. Pes GM, Errigo A, Soro S, Longo NP, Dore MP. Glucose-6-phosphate dehydrogenase deficiency reduces susceptibility to cancer of endodermal origin. Acta Oncol 2019;58(9):1205-11. Doi:10.1080/028418 6X.2019.1616815.

29. Karczewski KJ, Francioli LC, Tiao G, et al. The mutational constraint spectrum quantified from variation in 141,456 humans. Nature 2020;581(7809):434-43. Doi:10.1038/ s41586-020-2308-7.

30. Treviño LR, Shimasaki N, Yang W, et al. Germline genetic variation in an organic anion transporter polypeptide associated with methotrexate pharmacokinetics and clinical effects. J Clin Oncol 2009;27(35):59728. Doi:10.1200/JCO.2008.20.4156.

31. Ramsey LB, Bruun GH, Yang W, et al. Rare versus common variants in pharmacogenetics: SLCO1B1 variation and methotrexate disposition. Genome Res 2012;22(1):18. Doi:10.1101/gr.129668.111. 
32. Ingelman-Sundberg $M$, Mkrtchian $S$, Zhou $Y$, Lauschke VM. Integrating rare genetic variants into pharmacogenetic drug response predictions. Hum Genomics 2018;12(1):26. Doi:10.1186/s40246018-0157-3.

33. Lauschke VM, Ingelman-Sundberg $M$. Prediction of drug response and adverse drug reactions: From twin studies to Next Generation Sequencing. Eur J Pharm Sci 2019;130:65-77. Doi:10.1016/j. ejps.2019.01.024.

34. Tennessen JA, Bigham AW, O'Connor $T D$, et al. Evolution and functional impact of rare coding variation from deep sequencing of human exomes. Science 2012;337(6090):64-69. Doi:10.1126/science. 1219240

35. 1000 Genomes Project Consortium, Abecasis GR, Auton A, et al. An integrated map of genetic variation from 1,092 human genomes. Nature 2012;491(7422):5665. Doi:10.1038/nature11632.

36. Yu MHC, Chan MCY, Chung CCY, et al. Actionable pharmacogenetic variants in Hong Kong Chinese exome sequencing data and projected prescription impact in the Hong Kong population. PLoS Genet 2021;17(2):e1009323. Doi:10.1371/journal.pgen.1009323.

37. Runcharoen C, Fukunaga K, Sensorn I, et al. Prevalence of pharmacogenomic variants in 100 pharmacogenes among Southeast Asian populations under the collaboration of the Southeast Asian Pharmacogenomics Research Network (SEAPharm). Hum Genome Var 2021;8(1):7. Doi:10.1038/s41439-021-00135-z.

38. Zhu Y, Yin D, Su Y, et al. Combination of common and novel rare NUDT15 variants improves predictive sensitivity of thiopurine-induced leukopenia in children with acute lymphoblastic leukemia. 1. 2018;103(7):e293-e295. Doi:10.3324/haematol.2018.187658.

39. Liu C, Yang W, Pei D, et al. Genomewide Approach Validates Thiopurine Methyl- transferase Activity Is a Monogenic Pharmacogenomic Trait. Clin Pharmacol Ther 2017;101(3):373-81. Doi:10.1002/cpt.463.

40. Janssens ACJW, Deverka PA. Useless until proven effective: the clinical utility of preemptive pharmacogenetic testing. Clin Pharmacol Ther 2014;96(6):652-54. Doi:10.1038/clpt.2014.186.

41. Swen JJ, Nijenhuis M, van Rhenen M, et al. Pharmacogenetic Information in Clinical Guidelines: The European Perspective. Clin Pharmacol Ther 2018;103(5):795-801. Doi:10.1002/cpt.1049.

42. Relling MV, Evans WE. Pharmacogenomics in the clinic. Nature 2015;526(7573):34350. Doi:10.1038/nature15817.

43. Luczak T, Brown SJ, Armbruster D, Hundertmark M, Brown J, Stenehjem D. Strategies and settings of clinical pharmacogenetic implementation: a scoping review of pharmacogenetics programs. Pharmacogenomics 2021;22(6):345-64. Doi:10.2217/ pgs-2020-0181.

44. Rasmussen-Torvik LJ, Stallings SC, Gordon AS, et al. Design and anticipated outcomes of the eMERGE-PGx project: a multicenter pilot for preemptive pharmacogenomics in electronic health record systems. Clin Pharmacol Ther 2014;96(4):4829. Doi:10.1038/clpt.2014.137.

45. Roncato R, Dal Cin L, Mezzalira S, et al. FARMAPRICE: A Pharmacogenetic Clinical Decision Support System for Precise and Cost-Effective Therapy. Genes (Basel) 2019;10(4). Doi:10.3390/ genes10040276.

46. Cecchin E, Roncato R, Guchelaar HJ, Toffoli G, Ubiquitous Pharmacogenomics Consortium. Ubiquitous Pharmacogenomics (U-PGx): The Time for Implementation is Now. An Horizon2020 Program to Drive Pharmacogenomics into Clinical Practice. Curr Pharm Biotechnol 2017;18(3):204-209. Doi:1 0.2174/1389201018666170103103619.

47. van der Wouden $\mathrm{CH}$, Cambon-Thomsen $A$, Cecchin $E$, et al. Implementing Pharmacogenomics in Europe: Design and 
Implementation Strategy of the Ubiquitous Pharmacogenomics Consortium. Clin Pharmacol Ther 2017;101(3):341-58. Doi:10.1002/cpt.602.

48. Xiao Q, Zhou Y, Winter S, et al. Germline variant burden in multidrug resistance transporters is a therapy-specific predictor of survival in breast cancer patients.
Int J Cancer 2020;146(9):2475-2487. Doi:10.1002/ijc.32898.

49. Mukerjee G, Huston A, Kabakchiev B, Piquette-Miller $M$, van Schaik R, Dorfman R. User considerations in assessing pharmacogenomic tests and their clinical support tools. NPJ Genom Med 2018;3. Doi:10.1038/s41525-018-0065-4. 